\title{
Enhanced Fluorescence of Cy5-labeled Oligonucleotides near Silver Island Films (SIFs): A Distance Effect Study using Single Molecule Spectroscopy (SMS)
}

\author{
Yi Fu and Joseph R. Lakowicz*
}

Center for Fluorescence Spectroscopy, University of Maryland School of Medicine, Department of Biochemistry and Molecular Biology, 725 West Lombard Street, Baltimore, MD 21201

The time traces presented in Figure 2 were analyzed by preparing histograms of the emission count rates. Different levels of fluorescence signal are observed. The distributions shown in both Figure S-1 A and B depicts the "on" and "off" states via the appearance of one or two peaks.

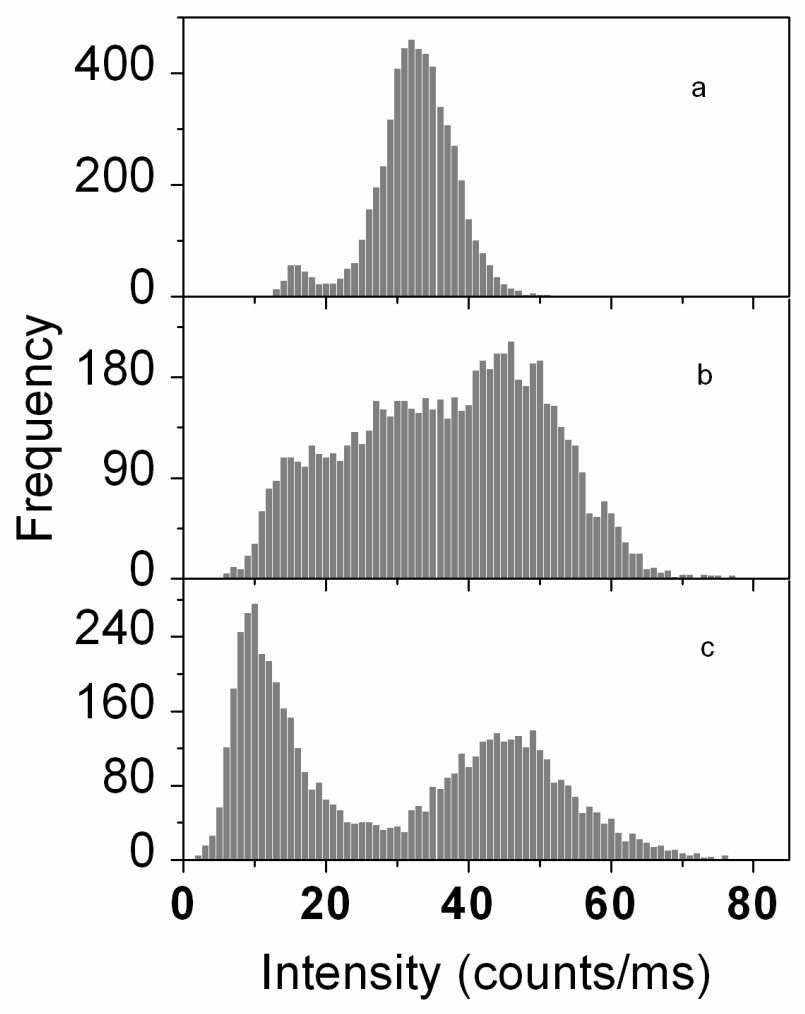

Figure S-1 A 


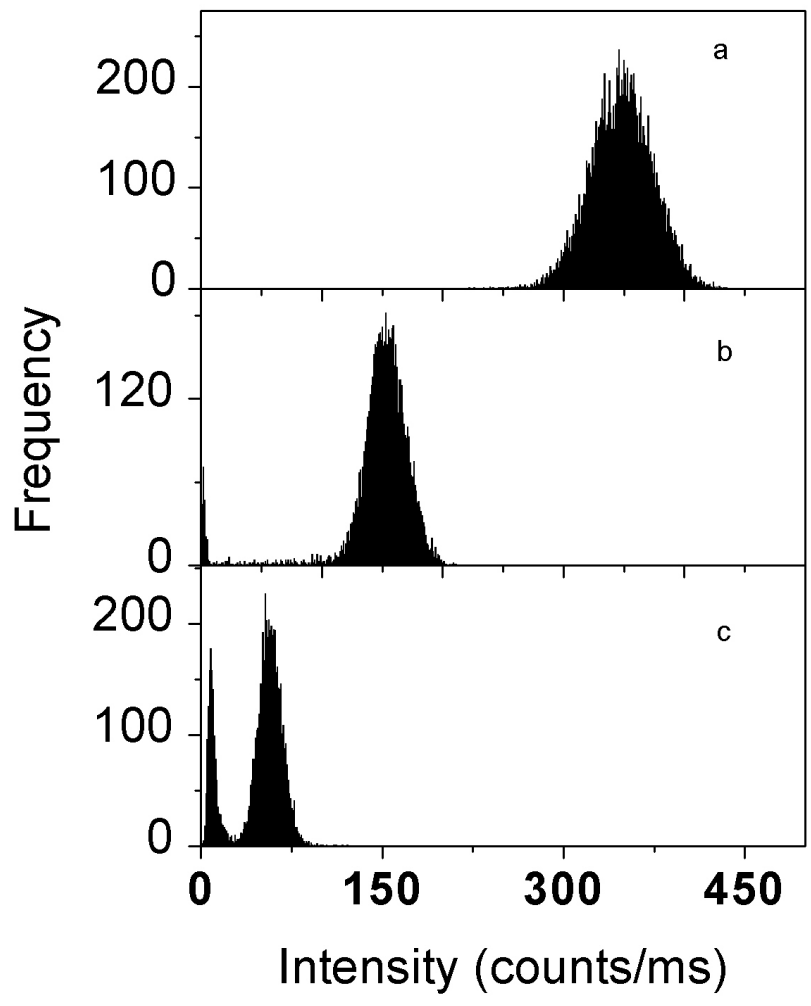

Figure S-1B

Figure S-1. Histograms of signal levels for the fluorescence time traces (derived from Figure 2 before photobleaching) on glass (A) and on SIFs (B), respectively. a: one BSA-biotin layer; b: double BSA-biotin layers; c: triple BSA-biotin layers. 


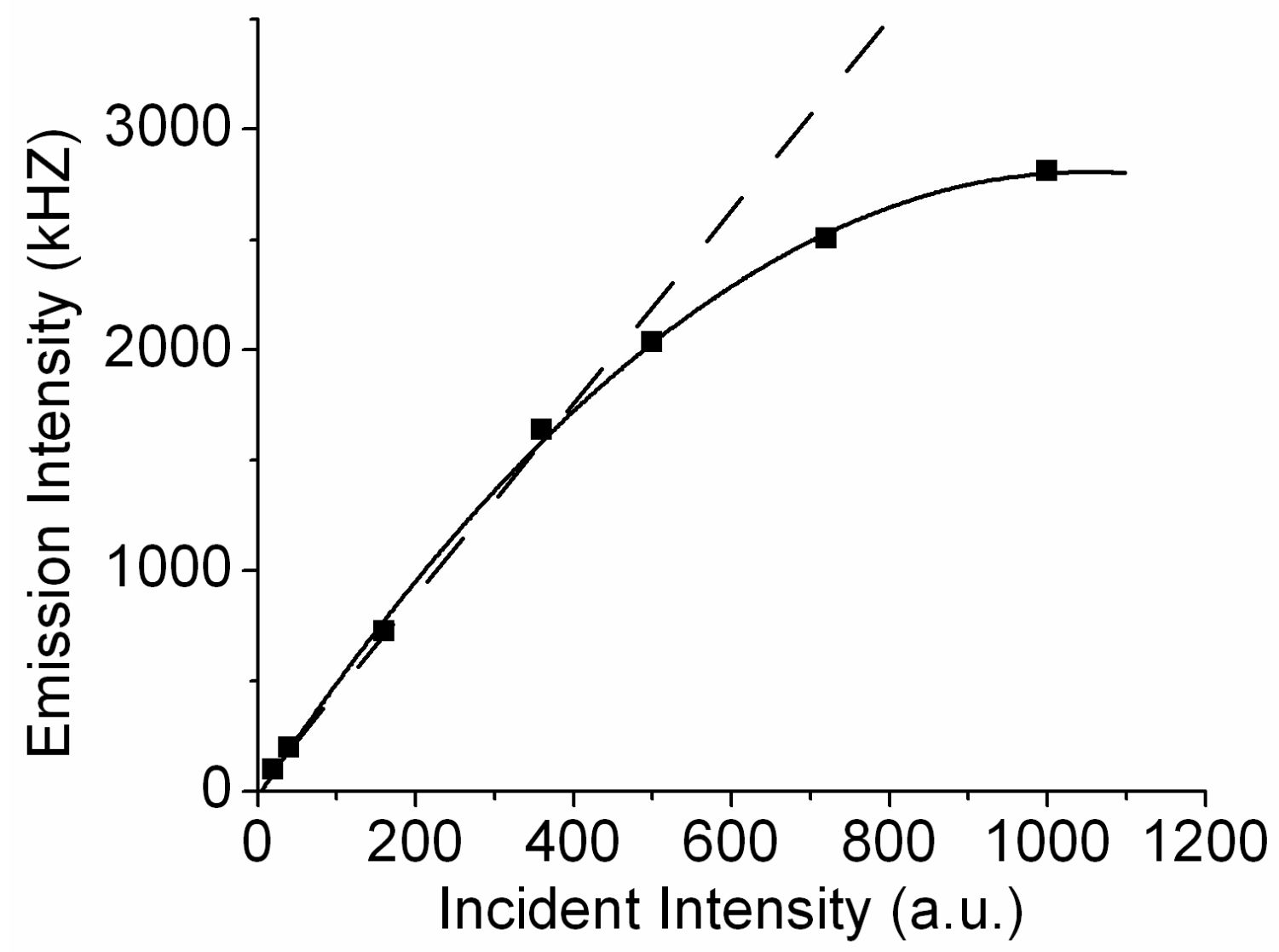

Figure S-2. Dependence of emission intensity on the incident laser power. 


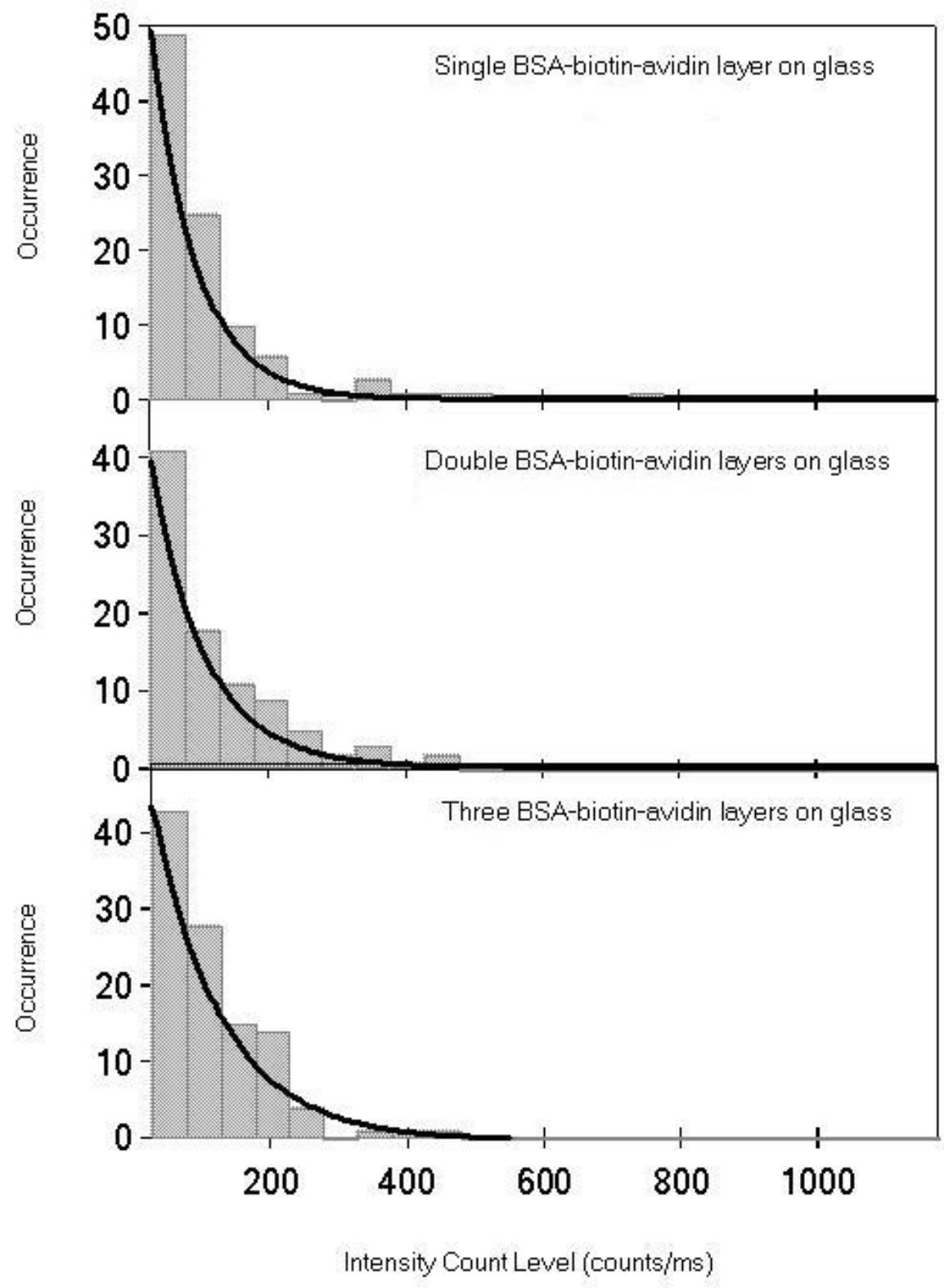

Figure S-3A Histograms showing distributions of fluorescence intensity levels recorded with various BSA-biotin-Avidin layers on glass substrates. 


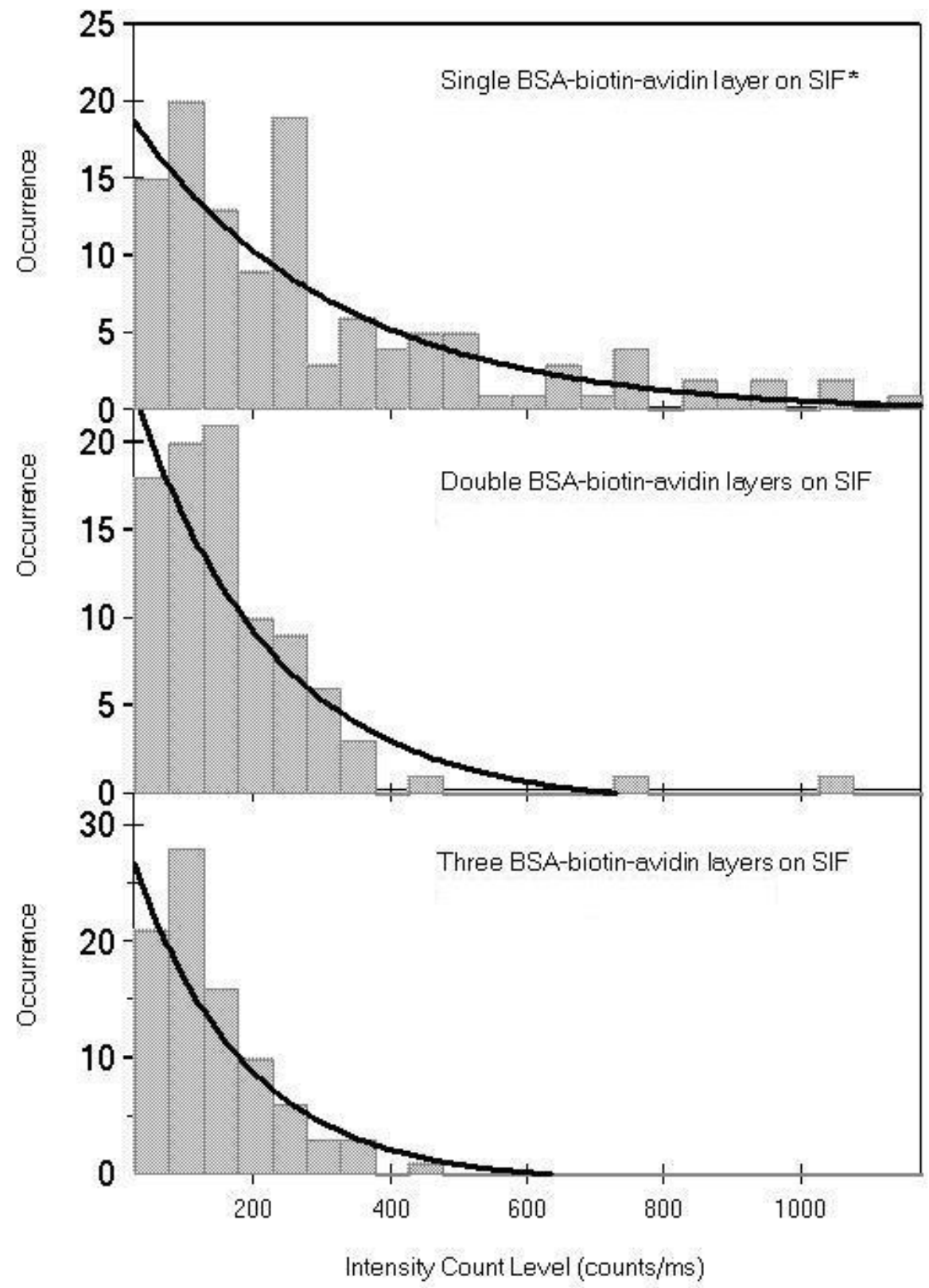

Figure S-3B Histograms showing distributions of fluorescence intensity levels recorded with various BSA-biotin-Avidin layers on silver island films. 